\title{
Erratum: Local Deterministic Model of Singlet State Correlations Based on Relaxing Measurement Independence [Phys. Rev. Lett. 105, 250404 (2010)]
}

Michael J. W. Hall

(Received 6 May 2016; published 26 May 2016)

DOI: 10.1103/PhysRevLett.116.219902

The degree of measurement dependence was incorrectly calculated for the singlet state model in the Letter, as $M_{\text {singlet }}=M_{\mathrm{CHSH}}$. Here

$$
M_{\mathrm{CHSH}}:=2(\sqrt{2}-1) / 3 \approx 0.276142
$$

is the minimum amount of measurement dependence required to model the maximum quantum violation of the $\mathrm{CHSH}$ inequality [1], as noted in the conclusion of the Letter.

The error arose from an incorrect algebraic maximization over the angles $\phi_{x y}$ and $\phi_{x^{\prime} y^{\prime}}=\phi^{\prime}$ defined in the Letter. The correct value of the measurement dependence may be calculated via a direct numerical maximization over these angles, yielding

$$
M_{\text {singlet }} \approx 0.276434 \text {. }
$$

This is approximately $0.1 \%$ higher than $M_{\mathrm{CHSH}}$ and corresponds to a choice of angles $\phi_{x y}=\phi_{x^{\prime} y^{\prime}} \approx 1.032 \pi / 4$.

While the magnitude of the error is tiny and does not change any of the central results of the Letter, it does open up the interesting possibility that there is a singlet state model with a degree of measurement dependence $M$ satisfying

$$
0.276142 \leq M<0.276434 .
$$

In particular, while it remains possible that the singlet state model in the Letter is optimal with respect to the degree of measurement dependence, it can currently be asserted to be optimal only to within $0.1 \%$.

I thank Cyril Branciard for pointing this error out to me and Andrew Friedman and Alan Guth for verifying the above numerical calculation of $M_{\text {singlet }}$.

[1] M. J. W. Hall, Phys. Rev. A 84, 022102 (2011). 